\title{
Improving Iterative Retinex Algorithm for Dynamic Range Compression
}

\author{
Shengdong Pan, Xiangjing An, Hongtao Xue, and Hangen He \\ College of Mechatronics Engineering and Automation \\ National University of Defense Technology \\ Changsha, Hunan, China \\ shdpan1984@gmail.com
}

\begin{abstract}
In this paper, the iterative Retinex algorithm is improved to handle dynamic range compression problems. Based on the analysis of the McCann-Sobel algorithm, the iterative smoothing operation can be interpreted as an asymmetric diffusion. In order to characterized the sharp discontinuities in illumination, an edge-stopping function is introduced into the iterative procedure, which is inspired by anisotropic diffusion. Using the improved illumination estimator, dynamic range of images can be arbitrarily manipulated while suppressing undesirable artifacts. Experiments show that the proposed algorithms outperforms several derivatives for dynamic range compression based on the iterative Retinex.
\end{abstract}

Keywords-iterative Retinex algorithm; anisotropic diffusion; dynamic range compression

\section{INTRODUCTION}

E. Land's Retinex theory addresses the problem that the human visual systems (HVS) can separate the reflectance $R$ of objects from the intensity $I$ of a given scene[9], [8], which can be formulated as:

$$
I=R * L
$$

where $L$ is the illumination of the scene. Since (1) is an illposed problem, it is impossible to be solved unless we have some prior knowledge about $R$ or $L$.

After decades' evolution, Retinex algorithms are now widely applied in many aspects of image processing, such as dynamic range compression, colored illumination correction, and general image enhancement, and so on[7], [13]. Despite the great number of variants, however, the basic assumption employed by Retinex is that edges in the scene are edges in the reflectance, whereas illumination changes slowly. Thus, in most Retinex algorithms, the estimated $L$ is a smooth version of the input $I$. Consequently, most of these algorithms may fail to produce persuasive results when processing images with abrupt illumination discontinuities, e.g. in compressing the dynamic range of images.

For computational convenience, the luminancereflectance model depicted in (1) is usually first converted into the logarithmic domain, such that:

$$
i=r+l
$$

where $i=\log (I), r=\log (R)$, and $l=\log (L)$. Thus the multiplicative problem becomes additive.

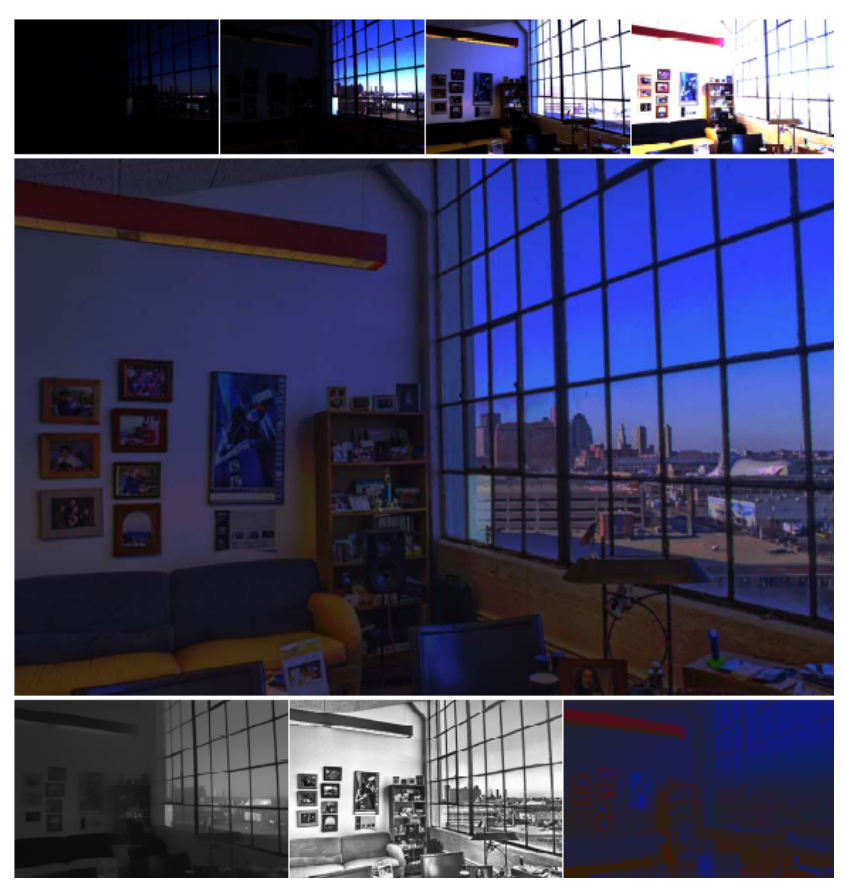

Figure 1. Window scene: an illustration of the proposed method. Top row: the original high dynamic range radiance map, from left to right, normalized, $\times 10, \times 100$, and $\times 1000$, respectively. Note that details all around the entire image can not be well represented in any single one of the four images. Middle row: the artifact-free result of dynamic range compression using the proposed method. Bottom row: the original high dynamic range radiance map is decomposed into three portions using the proposed method, the illumination, the reflectance, and the color, from left to right, respectively. For illustration purposes, the range of the illumination is reduced by $\gamma$ compression, where $\gamma=3$.

For a scene without direct light source, objects never reflect more energy than the incident light, such that:

$$
\forall p \in \Omega, l_{p} \geq i_{p} \text {, i.e. } r_{p} \leq 0
$$

Thus it is convenient to compute the illumination using the image upper envelope under the basic assumption.

In order to characterize the abrupt illumination discontinuities commonly associated with high dynamic range scenes, an edge-preserving illumination estimation algorithm is proposed in this paper. Based on the iterative Retinex algorithm proposed by McCann and Sobel in [10], we introduce an edge-stopping function into the iterative envelope estimation. Since this work is not intending to 
correct colored illumination, the original color image is first converted into an gray-scale image. Then this luminance component is further decomposed into colorless illumination and reflectance using the proposed algorithm, see Figure 1 for illustration. After reducing the range of the luminance component, the color information is reproduced to compose an image with similar color appearance to the original one. Experiments show that the proposed method is capable of modeling the discontinuities in illumination, and remove undesirable artifacts usually produced by standard Retinex algorithms.

\section{The ITERATIVE RetineX AlgORITHMS}

The first iterative Retinex algorithm is presented in a patent in [5], named after the authors, the Frankle-McCann Retinex algorithm. The iterative update operation of this algorithm can be formulated as follows:

$$
r_{p}^{k+1}=\frac{\operatorname{reset}\left(i_{p}-i_{q}+r_{q}^{k}\right)+r_{p}^{k}}{2}
$$

where $p$ is the neighborhood center pixel index, and $q$ is the index of one of the neighboring point. Let $p=(x, y)$, then $q \in\left\{\left(x \pm d^{k}, y\right),\left(x, y \pm d^{k}\right)\right\}$, where $d^{k}$ is the shift distance corresponding to the $k$-th update operation. In the iterative procedure, for a given $p, q$ is spirally taken, and $d^{k}$ is progressively reduced towards zero.

Equation (4) is known as the ratio-product-reset-average model for Retinex theory. Since the operations are all finished in logarithmic domain, the term $i_{p}-i_{q}$ is the ratio between the original intensity at $p$ and that at $q$. The following addition is the product operation. Then the ratioproduct term is reset to a constant whenever it exceeds the constant. And finally the reflectance estimation is updated by averaging the last estimation and the reset term. Equivalently, (4) can be rewritten as:

$$
r_{p}^{k+1}=\frac{\min \left\{c, i_{p}-i_{q}+r_{q}^{k}\right\}+r_{p}^{k}}{2}
$$

where $c=1$ in [6]. have:

Let $c=0$, and plug (2) into the above formulation, we

$$
\begin{aligned}
l_{p}^{k+1} & =i_{p}-r_{p}^{k+1} \\
& =i_{p}-\frac{\min \left\{0, i_{p}-l_{q}^{k}\right\}+i_{p}-l_{p}^{k}}{2}
\end{aligned}
$$

which can be further simplified as:

$$
l_{p}^{k+1}=\frac{l_{p}^{k}+\max \left\{i_{p}, l_{q}^{k}\right\}}{2}
$$

Note that (7) is exactly the formulation of the McCannSobel Retinex algorithm implemented in [10]. Instead of computing the reflectance directly from the original image in the Frankle-McCann algorithm, McCann and Sobel adopt the iterative scheme to first estimate the illumination.

Funt et al. generalize the Franckle-McCann algorithm to cope with arbitrary size of input images[6], where the effect of different parameter settings is discussed as well. An instructional discussion about the selection of the input parameters of the Frankle-McCann algorithm is also presented in [3].

Cooper and Baqai propose some extensions to the Frankle-McCann Retinex algorithm in [4], including introducing distance weighting, preferring dual spiral processing rather than single spiral processing, and suggesting a soft reset instead of the hard reset. The formulation with distance weighting can be given as:

$$
r_{p}^{k+1}=\frac{\operatorname{reset}\left(w\left(i_{p}-i_{q}\right)+r_{q}^{k}\right)+r_{p}^{k}}{2}
$$

Similarly in [14], based on the Frankle-McCann iterative procedure, Sobol introduces a ratio modification operator (RMO) into the update operation. The modified formulation can be given as:

$$
r_{p}^{k+1}=\frac{\operatorname{reset}\left(\delta_{t}\left(i_{p}-i_{q}, \alpha\right)+r_{q}^{k}\right)+r_{p}^{k}}{2}
$$

where

$$
\delta_{t}(z, \alpha)= \begin{cases}\operatorname{sgn}(z) * t & \text { if }|\alpha z|>t \\ \alpha z & \text { otherwise }\end{cases}
$$

where $\alpha$ is a contrast gain which controls the sharpness of the reproduced contrasts. The performance of the algorithm is further improved by varying $\alpha$ according to the shift distance, which is very similar to the distance weighting in [4]. Moreover, Sobol introduces a partial strength mask to render the strength of the dynamic range compression.

With these modifications, large contrasts are reduced, while small contrasts are preserved. These improvements are quite effective in dynamic range compression. However, such contrast truncation may lead to spatial distortions, as pointed out in [11].

\section{THE PROPOSED METHOD}

As proved in the previous section, when the ratio-product term is reset to 0, the Frankle-McCann algorithm is equivalent to the McCann-Sobel Retinex algorithm. Based on the latter algorithm in [10], we slightly modify the averaging operation to enable the preservation of edges in illumination estimation.

Removing the non-linear operation for the upper envelope (the max operator) in (7), we have:

$$
l_{p}^{k+1}=\frac{l_{p}^{k}+l_{q}^{k}}{2}
$$

A more explicit differential form of (11) can be given as follows:

$$
l_{p}^{k+1}-l_{p}^{k}=\frac{1}{2}\left(l_{q}^{k}-l_{p}^{k}\right)
$$

It is an asymmetric isotropic diffusion formulation, where only one neighboring point is taken into account during every update operation. To compensate the asymmetric behavior, the McCann-Sobel algorithm spirally takes four orthogonal directions while averaging.

The anisotropic diffusion equation proposed by Perona and Malik in [12] can be formulated as follows: 


$$
\frac{\partial I}{\partial t}=\operatorname{div}(g(\|\nabla I\|) \nabla I)
$$

whose discrete form is given as:

$$
I_{p}^{k+1}=I_{p}^{k}+\frac{\lambda}{\left|\eta_{p}\right|} \sum_{q \in \eta_{p}} g\left(\left|I_{p}-I_{q}\right|\right)\left(I_{p}-I_{q}\right)
$$

where $\lambda$ is the step size of the discrete time steps, with $0<\lambda \leq 1 . \eta_{p}$ denotes the spatial neighborhood of the pixel at $p$, and $\left|\eta_{p}\right|$ is the number of neighbors, (usually four for interior pixels). $g(\cdot)$ is the edge-stopping function.

Inspired by the discrete form of the anisotropic diffusion given in (14), we introduce a similar edge-stopping function into (12) to weight the asymmetric diffusion process. Thus, the modified edge-preserving local smoothing model is formulated as below:

$$
l_{p}^{k+1}-l_{p}^{k}=\frac{1}{2} g\left(\left|l_{q}^{k}-l_{p}^{k}\right|\right)\left(l_{q}^{k}-l_{p}^{k}\right)
$$

Similar to the formulation depicted in (11), the above equation can be further rewritten as below:

$$
l_{p}^{k+1}=\left(1-\frac{g\left(\left|l_{q}^{k}-l_{p}^{k}\right|\right)}{2}\right) l_{p}^{k}+\frac{g\left(\left|l_{q}^{k}-l_{p}^{k}\right|\right)}{2} l_{q}^{k}
$$

Now that (16) is an asymmetric derivative of anisotropic diffusion, combining it with (7), the proposed model can be formulated as follows:

$$
\begin{aligned}
l_{p}^{k+1}= & \left(1-\frac{g\left(\left|l_{q}^{k}-l_{p}^{k}\right|\right)}{2}\right) l_{p}^{k}+ \\
& \frac{g\left(\left|l_{q}^{k}-l_{p}^{k}\right|\right)}{2} \max \left\{i_{p}, l_{q}^{k}\right\}
\end{aligned}
$$

Consequently, an anisotropic diffusion based envelope estimation is obtained. The spatial behavior of the proposed model can be illustrated in Figure 2.

\section{DynAmic RANGe Compression Using The PROPOSED MODEL}

Now that the proposed algorithm is able to characterize the abrupt discontinuities in illumination, it is convenient to compress the dynamic range of an image without being disrupted by undesirable artifacts such as halos.

When handling color high dynamic range images, the original intensities in RGB color space is first converted into a gray-scale image. In our experiments, the following conversion model is applied.

$$
I=0.299 R+0.587 G+0.114 B
$$

Colorless illumination and reflectance of the image are then estimated using the proposed algorithm. Following the work in [12], the following edge-stopping function is chosen:

$$
g(x)=e^{-\frac{x^{2}}{2 \sigma_{r}^{2}}}
$$

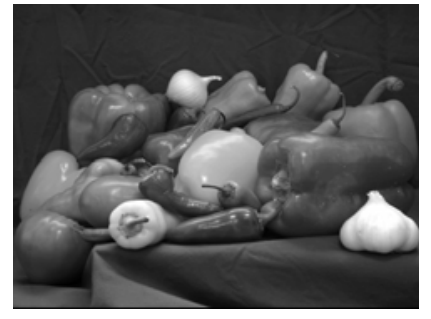

(a) test input

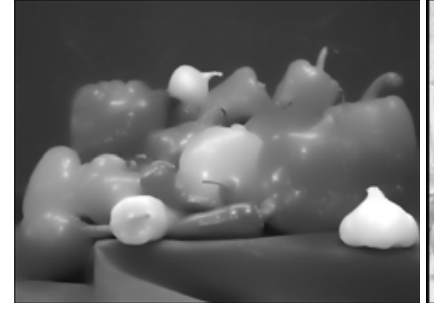

(b) the illumination

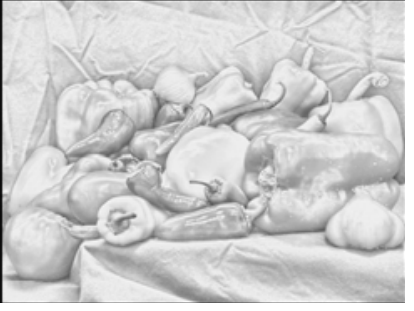

(c) the corresponding reflectance
Figure 2. The illumination + reflectance decomposition using the proposed method. Note that the sharp edges are preserved in the illumination, and the corresponding reflectance is free of halo artifacts.

After estimating the edge-preserving illumination, dynamic range compression is performed. The output intensity in logarithmic domain is calculated as follows:

$$
i^{*}=\frac{l}{\gamma_{1}}+\frac{r}{\gamma_{2}}
$$

where $\gamma_{1}$ corresponds to discounting the effect of the illumination, and $\gamma_{2}$ is introduced to enable the manipulation of the strength of the local contrast of the output image, and generally $1<\gamma_{2}<\gamma_{1}$. We find in experiments that the proposed algorithm produces good results with $\gamma_{1}=4.5$ and $\gamma_{2}=1.5$ in most cases.

Finally, the color information is reproduced according to the original high dynamic range radiance map, which is given as:

$$
C_{\text {out }}=\left(\frac{C_{\text {in }}}{I_{\text {in }}}\right)^{S} I_{\text {out }}
$$

where $C \in\{R, G, B\}$ denotes one of three independent color components in RGB color space. $I_{\text {in }}$ and $I_{\text {out }}$ denote the colorless intensities in linear domain before and after dynamic range compression, respectively. And $S$ is a parameter which controls the saturation of the reproduced color.

Then the proposed algorithm is compared with two other derivatives of the iterative Retinex algorithm for dynamic range compression, [14] and [11], respectively. In the experiment, four high dynamic range images are randomly selected from the existing high dynamic range radiance courtesies, as illustrated in Figure 3. In order to avoid 


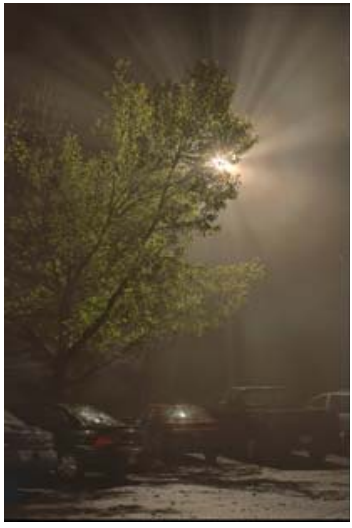

(a) Foggy night

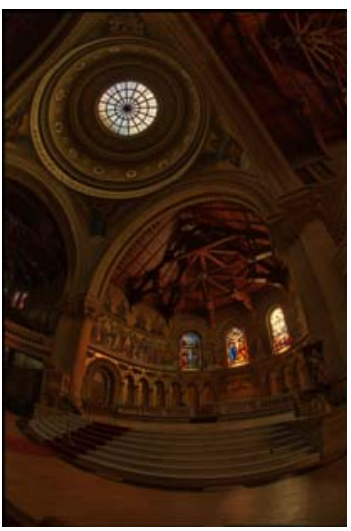

(c) Memorial

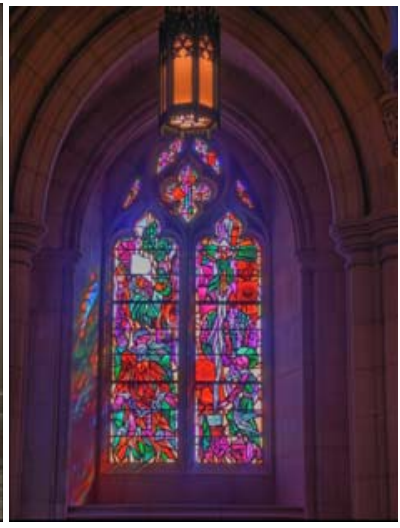

(b) Cathedral

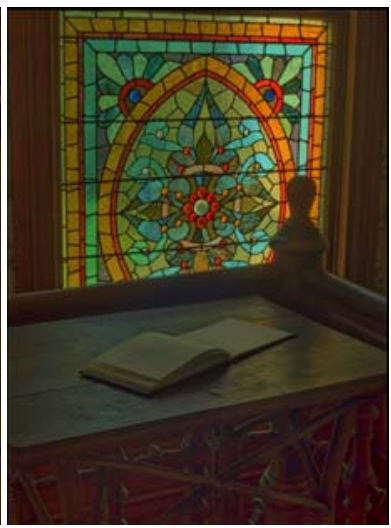

(d) Desk
Figure 3. Four high dynamic range radiance maps involved in the comparison is illustrated, which are produced by the proposed algorithm.

ambiguity of subjective evaluation, we measure the distortions in the result images quantitatively using the dynamic-range-independent image quality assessment metric proposed by Aydin et al. in [1]. The percentage of three types of distortions, contrast reversal $(E r)$, loss of visible contrast $(E I)$, and amplification of invisible contrast $(E a)$, respectively, and the average distortion error (Eavg) are listed in Table I. It is shown that the proposed algorithm produces less error than the counterparts.

\section{CONCLUSIONS}

The iterative image envelope estimation in McCannSobel Retinex algorithm is combined with the anisotropic diffusion in this paper. Thus the proposed algorithm is an edge-preserving envelope estimator. Such improvement is quite effective in dynamic range compression. Based on the decomposition of the original intensity, the dynamic range of an image can be arbitrarily manipulated, yet producing less visible differences.

\section{REFERENCES}

[1] T. O. Aydin, R. Mantiuk, K. Myszkowski, and H.-P. Seidel. Dynamic range independent image quality assessment. ACM Trans. Graph., 27(3):69:1-69:10, Aug. 2008.

[2] M. Black, G. Sapiro, D. Marimont, and D. Heeger. Robust anisotropic diffusion. Image Processing, IEEE Transactions on, 7(3):421 -432, mar 1998.

[3] F. Ciurea and B. V. Funt. Tuning Retinex parameters. J. Electronic Imaging, 13(1):58-64, 2004.

[4] T. J. Cooper and F. A. Baqai. Analysis and extensions of the FrankleMccann Retinex algorithm. J. Electronic Imaging, 13(1):85-92, 2004.

[5] J. A. Frankle and J. J. Mccann. Method and apparatus for lightness imaging. U.S. patent, (4384336), May 1983.

[6] B. Funt, F. Ciurea, and J. McCann. Retinex in MATLAB, Journal of Electronic Imaging, 13(1):48-57, 2004.

[7] D. Jobson, Z. Rahman, and G. Woodell. A multiscale retinex for bridging the gap between color images and the human observation of scenes. Image Processing, IEEE Transactions on, 6(7):965 -976, jul 1997.

[8] E. H. Land. Recent advances in retinex theory and some implications for cortical computations: color vision and the natural image. Proceedings of the National Academy of Sciences, 80(16):5163-5169, 1983.

[9] E. H. Land and J. J. McCann. Lightness and the retinex theory. Journal of Optic Society of American, 61:1-11, 1971.

[10] J. J. McCann and I. Sobel. Experiments with Retinex. HPL Color Summit, 1998.

[11] S.-D. Pan, X.-J. An, and H.-G. He. Combining recursive retinex and eye adaptation for high dynamic range compression. In Wavelet Analysis and Pattern Recognition (ICWAPR), 2011 International Conference on, pages $115-120$, july 2011.

[12] P. Perona and J. Malik. Scale-space and edge detection using anisotropic diffusion. Pattern Analysis and Machine Intelligence, IEEE Transactions on, 12(7):629 -639, jul 1990.

[13] D. Shaked and R. Keshet. Robust recursive envelope operators for fast retinex. Technical report, 2002.

[14] R. Sobol. Improving the retinex algorithm for rendering wide dynamic range photographs. Journal of Electronic Imaging, 13(1):6574, 2004.

TABLE I. PERFORMANCE COMPARISON

\begin{tabular}{|c|c|c|c|c|c|c|c|c|c|c|c|c|}
\hline \multirow{2}{*}{ image } & \multicolumn{4}{|c|}{ the proposed method } & \multicolumn{4}{|c|}{ [14] } & \multicolumn{4}{|c|}{ [11] } \\
\hline & Er & EI & $E \boldsymbol{E}$ & Eavg & $\boldsymbol{E r}$ & El & $\boldsymbol{E} \boldsymbol{a}$ & Eavg & $\boldsymbol{E r}$ & El & $\boldsymbol{E} \boldsymbol{a}$ & Eavg \\
\hline Foggy night & 2.21 & 4.03 & 0.59 & 2.28 & 12.45 & 3.51 & 58.76 & 24.91 & 5.04 & 3.46 & 37.61 & 15.37 \\
\hline Cathedral & 11.87 & 15.12 & 4.94 & 10.64 & 18.94 & 28.03 & 29.32 & 25.43 & 16.63 & 16.88 & 35.78 & 23.10 \\
\hline Memorial & 8.92 & 18.86 & 0.60 & 9.46 & 19.67 & 21.83 & 11.17 & 17.56 & 17.68 & 5.84 & 14.63 & 12.72 \\
\hline Desk & 12.03 & 31.26 & 1.23 & 14.84 & 21.32 & 50.41 & 4.22 & 25.31 & 28.92 & 12.64 & 23.72 & 21.76 \\
\hline
\end{tabular}

